\title{
Small-World Reconfigured CAN for Distributed Control Plane of Future Optical Networks
}

\author{
Marconi Pereira Fardin, Moises R. N. Ribeiro, and Helio Waldman
}

\begin{abstract}
The transport layer structure of the Internet is moving towards a model of high-speed routers interconnected by intelligent optical core networks. A consensus has been reached on wavelength division multiplexing (WDM) as the way to increase the transmission capacity. However there are few proposals for distributed control planes under dynamic Quality of Service (QoS) constraints for large networks. In this context, resources availability must be frequently distributed and updated across the network; and flooding may no longer be the best strategy for information dissemination. This paper proposes and assesses an overlay structure based on distributed hash tables (DHT) for information indexing over bi-dimensional virtual concatenated spaces (VCS). By adding long-distance interconnections to Content-Addressable Networks (CAN), the resulting smallworld effect can reduce the number of hops per resource search. Analytical results indicate significant reduction in management traffic over the physical layer of large networks.
\end{abstract}

Keywords-Distributed Optical Control Plane, DHT, SmallWorld

\section{INTRODUCTION}

The widespread use of the Internet in recent years has led to a multitude of new services emerging from the IP (Internet Protocol) networks. For example, VoIP (Voice over IP), electronic commerce, file sharing, and IP-TV are becoming very popular among domestic users. The best-effort based networks of today can somehow handle such applications them. However, specific demands for reliable communications from the business community and the interactive real-time services pose more challenging problems to next generation networks. In other words, it is expected from future networks higher standards of Quality of Service (QoS) well beyond the present best-effort. There are indeed two bottlenecks to be addressed in order to meet tomorrow's networks demands, namely; i) bandwidth capacity at the transport infrastructure; and ii) the network operation and management (OAM) capability.

In order to cope with the Internet transport infrastructure bottleneck, a model of high-speed routers interconnected by optical core networks is being adopted [1]. The second generation of optical networks expands the wavelength division Multiplexing (WDM) capacity beyond the purpose of pointto-point interconnection. Networking functionalities, such as routing of transparent lightpaths across the physical topology,

Marconi Pereira Fardin, Department of Communications, School of Electrical and Computer Engineering, University of Campinas. Email: fardin@decom.fee.unicamp.br. Moises R. N. Ribeiro, Department of Electrical Engineering, Federal University of Espirito Santo. E-mail: moises@ele.ufes.br. Helio Waldman, Center for Engineering, Modeling and Applied Social Science, Federal University of ABC. E-mail: helio.waldman@ufabc.edu.br. This work is partial financed by Fapesp in process 2005/03836-6. are already feasible. However, the control plane standardization to integrate optical and electronic networks is lagging behind. The complexity of managing such a large number of resources and parameters in a dynamic way is the main challenge for control planes. Indeed, a transparent lightpath in a WDM optical network is an end-to-end tunnel spanning over several physical links (that usually goes along on a single wavelength). Optical Cross Connects (OXCs) are able to switch a wavelength from an incoming to an outgoing fiber while reconfigurable optical add-and-drop multiplexes (ROADMs) connect a given number of optical transceivers to the electronic layer of the network while other wavelengths pass by this node without being electronically processed.

OAM with automatic provisioning requires that the routing and wavelength assignment (RWA) problem to be dynamically solved for each lightpath connection request. The basic objective of a RWA algorithm is to optimize the network performance in terms of reducing blocking probability for lightpath requests. There are many RWA algorithms proposed in literature [2], [3], [4]. However there are scalability problems due to the requirement of global knowledge of network state. It is not yet a consensus whether centralized, distributed or even augmented approaches should be used to manage the information needed to provisioning viable route across the optical network [5]. Moreover, RWA integration with QoS (from both physical and electronic layers) will complicate even further the OAM problem in dynamic networks.

It is well-known that the resilience and scalability of present-day Internet is only achieved thanks to its distributed processing and decision process. The link state advertisement (LSA) sent by individual routers to their neighbors are used for optimal routing calculations in a distributed way. However, this information is periodically disseminated by flooding. In order, this is done to keep state consistency among nodes and whenever there is a state change as in open shortest path first (OSPF) protocol [6].

The addition of QoS dynamic parameters to LSAs in dynamic networks may lead to endless storms of management information being exchanged by nodes. This may be responsible for taking up large amounts of network bandwidth capacity. Thus, flooding will be no longer the most appropriated way of disseminating information in such future scenarios of dynamic networks. More scalable mechanism are yet to be proposed. However, the success of peer-to-peer (P2P) overlay networks might offer hints on how to evolve the control plane of large networks for to storing information and conducting searches in a distributed way.

The P2P systems became popular with file sharing applications, such as Napster [7] and Gnutella [8], because of their 
large capacity of information distribution. The centralization of Napster was, simultaneously, its the principal advantage and its main handicap. The evolution of P2P systems towards distributed approaches first relied on flooding. In distributed indexing with flooding architecture (DIFA), e.g., Gnutella, the content request are replicated to every neighbor node until the sought information is found. However, the flooding mechanism then became impracticable for the worldwide Internet scale.

A solution to this problem is the distributed indexing with hash architecture (DIHA). In this system information is evenly stored in the network nodes but it is done in such a way that the content request can be routed directly to the point of storage without the need of using the flooding mechanism. This is implemented by Distributed Hash Table (DHT) [9] over different overlay interconnection topologies such as Pastry [10], Chord [11], and content addressable network (CAN) [12]. In this paper we propose the use of small-world reconfigurations over CAN overlay structures as a template for distributed control plane in optical networks.

The remainder of this paper is organized as follows: Section II describes related works and the main contributions of this paper. Section III illustrates the architecture for storage and search over a structured virtual coordinated space. Section IV proposes the deterministic reconfiguration of the structured overlay topology in order to take advantages of the smallworld characteristics of social networks. In Section V it is carried out management traffic estimation for flooding based approach, overlay CAN, and overlay small-world reconfigured CAN topologies. Section VI presents the results and, finally, Section VII presents the concluding remarks.

\section{Related Works And PAPER CONTRibutions}

Provided that QoS oriented constraint-open shortest path first (CSPF) and its extensions to traffic engineering (OSPFTE) keep flooding mechanism as the means of distributing information across the network [13], they are bound to suffer from the LSA $n$-squared limitation [14]. A link state change in a $n$-node network requires $n^{2}$ LSA messages to be spread. Some authors try to cope with this problem by reducing the amount of flooding messages, e.g., [15], [16], [17]. However, only marginal improvements may be achieved and the reduced (and out-to-date) access to information may lead network performance to be impaired and, as a result, the QoS goal not to be met.

Only recently the similarities between $\mathrm{P} 2 \mathrm{P}$ applications and control-plane networks have been realized. P2P approach to control plane through a layered architecture using a SIP (section initialization protocol)-based control/signaling plane over Chord topology has been proposed in [18]. However, no additional information for QoS purposes are involved and control plane scalability issues are not addressed. Moreover, Chord is an one-dimension topology in which the number of hops and connections per node increases with the number of elements in the network. As a result, this solution might not be suitable for reducing management information across large networks.

The main contribution of this paper is the proposal of a deterministic small-world reconfiguration following [19] over a bi-dimensional (CAN) structured semantic space for noncentralized information distribution in large networks. An analytical assessment based on the total number of management packet over the physical topology is presented using as parameters the number of nodes, and number of resources to be advertised per node. This allows us to check flooding mechanism against bi-dimensional overlay architectures, with small-world reconfiguration.

\section{Proposed SOLUTION}

This paper intends to use DHT mechanism to develop a distributed control plane for optical networks, where large amounts of information have to be exchanged due to physical and electronic layers limitations. The control plane first has to evolve from DIFA-like structure to DIHA. In order to do so, the paradigm of information distribution must be reversed. The information to perform tasks such as bandwidth reservation, routing, and wavelength assignment must be fetched by nodes instead of being distributed regardlessly. The problem now is how to find information in a completely distributed network. The publication of network state in form of semantic codes, in a virtual coordinated space (VCS), is the proposed solution.

A hash coded resource state (e.g., a wavelength on a given fiber, the spare bandwidth in ongoing lightpaths, a wavelength converter etc.,) along with an identifier (IP or MAC addresses) of the publisher node is then let available in the VCS at the position indicated by their hash code. This enables the search for resources in the physical networks to be conducted in the virtual space by a node in need of serving a given connection request. Geometrically, the VCS is actually a torus but it is usually displayed using a bi-dimensional representation, which is turned into a torus when the opposite edges are connected.

The semantic codes are obtained through a hash function over a string with resource name. This function $(Z=h(s))$ is common to all nodes in the network and it has multiple purposes. First the physical node has to register itself using the hash function to codify its name. The location of resources that should published in the VCS is also given by using this hash function codification. When performing searches, a node makes use of the hash function to know the position in which the identifier of a required resource is stored in VCS. Therefore, in each position of the VCS will be held at most one resource identifier, so that functions like SHA [20] with collision resolution techniques are preferable.

The VCS will be divided among all registered nodes of the network. Each node will hold a range of semantic codes and identifiers. In order to access tables from different nodes, a overlay network is built through transmission control protocol (TCP) sessions between the overlay nodes. These connections reunify the VCS and make available information stored in particular overlay nodes. A more detailed illustration for the whole process will be given below.

\section{A. Virtual Structure Construction}

Starting from the physical network illustrated in Figure 1, each node generates its own hash code (in this example based 
on their names). Then they are registered in the VCS by notifing the node holding their hash code. After this registration process, the nodes should divide the VCS among them. Figure 2 illustrates this division. Note that a node can occupy larger chunks than others (e.g., $E$ ). Then the overlay network is established through neighbor relationships generated in VCS as shown in Figure 3. Each node establish $g_{o}\left(g_{o}=4\right.$ in Figure 3) interconnections in this overlay network. This are TCP sessions, which make use of the physical topology in Figure 1 to carry their packets.

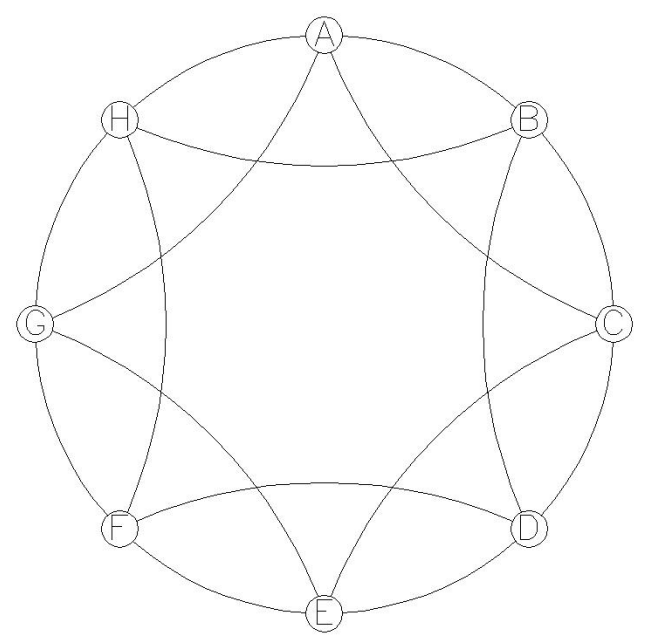

Fig. 1. Physical Network

\begin{tabular}{|c|c|c|}
\hline $\begin{array}{c}\text { Node G } \\
\text { area }\end{array}$ & $\begin{array}{c}\text { Node B } \\
\text { area }\end{array}$ & $\begin{array}{c}\text { Node F } \\
\text { area }\end{array}$ \\
\hline $\begin{array}{c}\text { Node E } \\
\text { area }\end{array}$ & $\begin{array}{c}\text { Node E } \\
\text { area }\end{array}$ & $\begin{array}{c}\text { Node C } \\
\text { area }\end{array}$ \\
$\begin{array}{c}\text { Node A } \\
\text { area }\end{array}$ & $\begin{array}{c}\text { Node D } \\
\text { area }\end{array}$ & Node H \\
area
\end{tabular}

Fig. 2. VCS division

\section{B. Walking in the Virtual Coordinated Space}

The routing across overlay networks is a very important issue to the success of the present proposal. This procedure should not produce much management traffic since it has to be transported across the physical layer. Moreover, a node must find the information about a given resource as fast as possible to quickly compute, for example, the best available route for a lightpath request. Resource state update should also be swift in order to avoid stale information being used in routing. By using the hash coded resource name, the requisition is forwarded node by node until it arrives to the one in charge

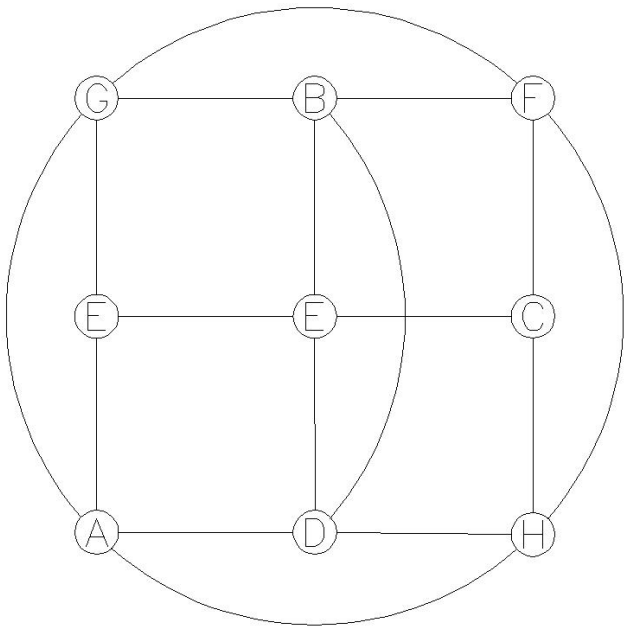

Fig. 3. Overlay Network

of holding this hash code in the node VCS area, or in physical terms: in its hash table stored in its memory.

Whenever a node receives a requisition, first it verifies if the requisition hash code lies in its hash table. If it is not the case, the requisition is then forwarded to the neighbor with the shortest (euclidian) distance from the hash code in VCS. However, before searching for (hash coded) information, those network resources must be registered across node regions in the VCS as follows.

\section{Semantic Space Occupation}

After performing nodes registry and overlay network setup via TCP sessions, the nodes can publish their physical resources. In Table I, it is illustrated few physical resources that are interconnecting nodes (transceivers on a given wavelength) and their corresponding hash codes $(x, y)$ for a particular hash function $h(s)$.

TABLE I

RESOURCE CODES

\begin{tabular}{|c|c|c|}
\hline Item & Resources $(s)$ & Coordinates $((x, y))$ \\
\hline \hline 1 & Wavelength $\lambda_{1}, 10 \mathrm{~Gb} / \mathrm{s}, \mathrm{A}-\mathrm{B}$ & $(12,01)$ \\
\hline 2 & Wavelength $\lambda_{1}, 10 \mathrm{~Gb} / \mathrm{s}, \mathrm{A}-\mathrm{C}$ & $(15,05)$ \\
\hline 3 & Wavelength $\lambda_{1}, 10 \mathrm{~Gb} / \mathrm{s}, \mathrm{A}-\mathrm{H}$ & $(05,04)$ \\
\hline 4 & Wavelength $\lambda_{1}, 10 \mathrm{~Gb} / \mathrm{s}, \mathrm{A}-\mathrm{G}$ & $(09,15)$ \\
\hline 5 & Wavelength $\lambda_{1}, 10 \mathrm{~Gb} / \mathrm{s}, \mathrm{B}-\mathrm{C}$ & $(14,13)$ \\
\hline 6 & Wavelength $\lambda_{1}, 10 \mathrm{~Gb} / \mathrm{s}, \mathrm{B}-\mathrm{D}$ & $(02,04)$ \\
\hline 7 & Wavelength $\lambda_{1}, 10 \mathrm{~Gb} / \mathrm{s}, \mathrm{C}-\mathrm{D}$ & $(12,06)$ \\
\hline 8 & Wavelength $\lambda_{1}, 10 \mathrm{~Gb} / \mathrm{s}, \mathrm{C}-\mathrm{E}$ & $(15,08)$ \\
\hline 9 & Wavelength $\lambda_{1}, 10 \mathrm{~Gb} / \mathrm{s}, \mathrm{H}-\mathrm{G}$ & $(09,11)$ \\
\hline 10 & Wavelength $\lambda_{1}, 10 \mathrm{~Gb} / \mathrm{s}, \mathrm{H}-\mathrm{F}$ & $(05,12)$ \\
\hline 11 & Wavelength $\lambda_{1}, 10 \mathrm{~Gb} / \mathrm{s}, \mathrm{G}-\mathrm{F}$ & $(13,02)$ \\
\hline 12 & Wavelength $\lambda_{1}, 10 \mathrm{~Gb} / \mathrm{s}, \mathrm{G}-\mathrm{E}$ & $(01,03)$ \\
\hline
\end{tabular}

For example, node $A$ will publish the resource 01 of the Table I, which corresponds to the resource code $(12,01)$, at the corresponding $(x, y)$ coordinates. The process is performed as follows. First node $A$ verifies if this resource is within its management area showed in Figure 2. As this is not the case, node $A$ forwards this resource to its closest neighbor in $V C S$. The requisition arrives on node $H$ (remember that the VCS 
is actually a torus). In this case the coordinates $(12,1)$ will match node $H$ area. Thus, it will be published in node $H$ hash table, as shown in Figure 4. Note that a link state change will produce modifications just to one node database, instead of the whole network being affected as in flooding-based schemes.

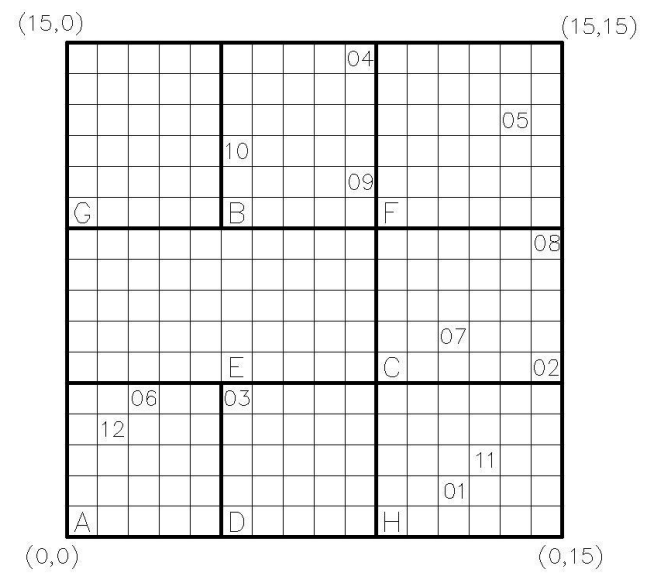

Fig. 4. Published Resources

\section{Deterministic Small World Virtual Space RECONFIGURATION}

For large networks the overlay routing process described above may become lengthy and inefficient as the number of hops increases in the VCS. A small-world reconfiguration of the overlay network has been considered as a means of reducing the mean number of hops between the overlay nodes by the addition of "long distance" connections [21], [22]. This concept comes from social network researches conducted in late 1960s [23]. There is a surprisingly small number of common acquaintances in between any two individuals across large geographical distances. This effect is basically down to the fact that people usually have at least few long distance relationships, and these few connections can help bypassing many intermediate hops. However, just a decade ago this concept was introduced by Watts and Strogatz as a model for explaining (randomly constructed) electrical distribution networks, the spreading of diseases in the population and many others networking including the Internet router interconnections [24].

This paper proposes a deterministic reconfiguration where some nodes $(C)$ are elected as hubs. They are interconnected by a partial mesh as described in Equation 2. Hub nodes will provide long distance connections to ordinary nodes. The number of required hubs are deterministically calculated from a parameter $d$, that represents the maximum distance between a node and a hub in the VCS. Figure 5 shows a large overlay network with 144 areas where hub where elected for $d=3$ and are represented by filled symbols. The connections between nodes, which are visible in Figure 3, have been here omitted for the sake of clear visualization. The lines represent the region of influence of hubs, i.e., nodes within this area will use a particular hub for long distance connections in the VCS, reducing overlay network diameter as a result. The resulting number of hubs $C$ in the overlay network for a given $d$ is calculated by Equation 1, where $n$ is the number of nodes in the overlay network (approximately the number of nodes in the physical network).

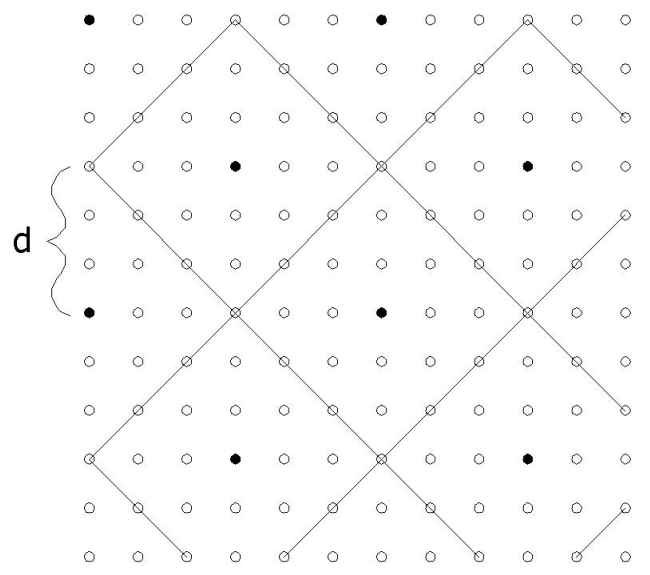

Fig. 5. Hubs election (Black Nodes)

$$
C=2 \times\lceil\sqrt{n} / 2 d\rceil^{2}
$$

Each hub node, say $i$, will hold a set of $V-g_{o}$ long distance connections to others hubs, indexed by $j$, according to Equation 2.

$$
\begin{aligned}
& j=\bmod \left(i+1+\left\lfloor k \times \frac{C-1}{V-g_{o}}\right\rfloor ; C\right), k=0,1,2, \ldots,\left(V-g_{o}-1\right) \\
& j, i \in\{0,1,2, \ldots, C-1\}
\end{aligned}
$$

\section{Analytical Methodology of Assessment}

In order to analytically evaluate the proposed control plane information distribution technique, a regular physical network topology (as the one in Figure 1) has been assumed. Irregular networks will possiblyrequire numerical simulations for assessing their performance. The number of nodes $(n)$ has been used as a parameter but with fixed physical degree $\left(g_{f}=4\right)$. The average number of hops in the physical network is given in Equation 3.

$$
H_{P N}=\left\lceil\frac{\lceil n / 2\rceil}{g_{f}}\right\rceil
$$

A static overlay network has been assumed for the sake of simplicity. The average number of hops in overlay network, with $\left(H_{O N_{S W}}\right)$ and without $\left(H_{O N_{G}}\right)$ small-world reconfiguration, are given by Equations 4 and 5, respectively.

$$
H_{O N_{S W}}=2\left\lceil\frac{\left\lceil\frac{\left\lceil\frac{2 d+1}{3}\right\rceil+\left(\left\lceil\frac{C}{V-g_{o}}\right\rceil+2\left\lceil\frac{2 d+1}{3}\right\rceil\right)(C-1)}{C}\right\rceil}{H_{O N_{G}}=2\left\lceil\frac{N}{g_{o} / 2}\right\rceil}\right.
$$

The average number of packets in the physical layer is evaluated for both Setup Traffic and Operational Traffic as described below. For Equations 6 to $12 H_{O N}$ accounts for hops in the overlay network and it is replaced by either Equation 4 for overlay networks with small-world reconfiguration or Equation 5 for classical VCS, which will be identified now on as "structured". 


\section{A. Setup Traffic $\left(T_{S}\right)$}

- node registration (NR): the node has to send a registration request to a neighbor already in the VCS. This request walks through the overlay network (bear in mind that packets will go along the physical network) until its find the point of insertion. The node in charge reply (ACK) the request to the requesting node. The amount of packets will then be:

$$
N R=1+H_{O N} \times H_{P N}+H_{P N}
$$

- neighbor setup (NS): the node contact its $V$ neighbors and exchange three packets to setup a TCP connection (i.e., $S I N, S I N+A C K$, and $A C K$ ). Therefore, the contribution to the Setup Traffic will be:

$$
N S=V \times 3 H_{P N}
$$

- resource publication (RP): the node has to send a publication request for each physical resource to be published $\left(N_{R P}\right)$ to the overlay network. Then, an acknowledgement, from the node in charge of each item is sent back to the node that holds the physical resource.

$$
R P=N_{R P} \times H_{P N}\left(H_{O N}+1\right)
$$

Finally, the total average Setup Traffic per node given by Equation 9.

$$
\begin{array}{ccc}
T_{S} & = & N S+N R+R P \\
& = & 1+H_{P N}\left[H_{O N}\left(1+N_{R P}\right)+3 V+N_{R P}+1\right]
\end{array}
$$

\section{B. Operation Traffic $\left(T_{O}\right)$}

- resource fetching (RF) for each resource fetched (from a set with $N_{R F}$ ) the fetching node has to send a fetch request to the VCS areas holding the fetched resource. The response will follow the request and an acknowledgment is sent back to the node in charge of holding the information. The total number of packets will then be:

$$
R F=H_{P N}\left(H_{O N} N_{R F}+2 N_{R F}\right)
$$

- resource updating (RU) similar to resource fetching, resource update follows a identical procedure except for the last acknowledgement that is sent by the node in charge of holding the information. Thus the number of packets generated by $N_{R U}$ updates is:

$$
R U=H_{P N}\left(H_{O N} N_{R U}+N_{R U}\right)
$$

Finally, the total average Operational Traffic is:

$$
\begin{array}{ccc}
T_{O} & = & R F+R U \\
& = & H_{P N}\left[H_{O N}\left(N_{R F}+N_{R U}\right)+2 N_{R F}+N_{R U}\right]
\end{array}
$$

\section{Optimal Neighbor Number}

The optimal number of neighbors in a small-world reconfiguration that minimizes the amount of packets can be then found by differentiating the sum of equations 9 and 12 . The optimal $V_{o p}$ is then presented in equation 13.

$$
V_{o p}=g_{o}+\sqrt{\frac{2(C-1)\left(1+N_{R P}+2 N_{R F}+N_{R U}\right)}{3}}
$$

\section{Flooding}

The corresponding number of packets when using the flooding mechanism for Setup Traffic, is computed as: $n$ nodes flooding the network with items through all ports (excluding the one it first received the flooding message). There is also an acknowledgment message. Therefore the Setup Traffic can be stated in Equation 14. Regarding the amount of Operation Traffic, where a information needs to be updated across the networks nodes (with acknowledgment), is presented by Equation 15.

$$
\begin{gathered}
T_{S_{F}}=n \times N_{R P} \times g_{f} \times n \times 2 \\
T_{O_{F}}=N_{R U} \times g_{f} \times n \times 2
\end{gathered}
$$

\section{RESUlts}

Results are aimed at comparing flooding mechanism with structured VCS and small-world reconfigured VCS under different values for $d$ parameter in different scenarios. The amount of packets (information exchanged $\times$ number of hops) in the physical layer is the adopted metric. Moreover, the amount of traffic is presented in terms of Setup Traffic to build the VCS and Operation Traffic.

In Figure 6 the effect of network growth on the Setup Traffic is presented for $N_{R P}=128, N_{R F}=0$, and $N_{R U}=0$. It is clear that the proposed mechanism can significantly (nearly one order of magnitude) reduce the Setup Traffic when compared with flooding. However, small-world reconfiguration becomes more important for larger networks (beyond $10^{3}$ nodes), where performance is more sensitive to $d$. It is noteworthy that $d=3$ brings a good performance for whole range number of nodes in Figure 6.

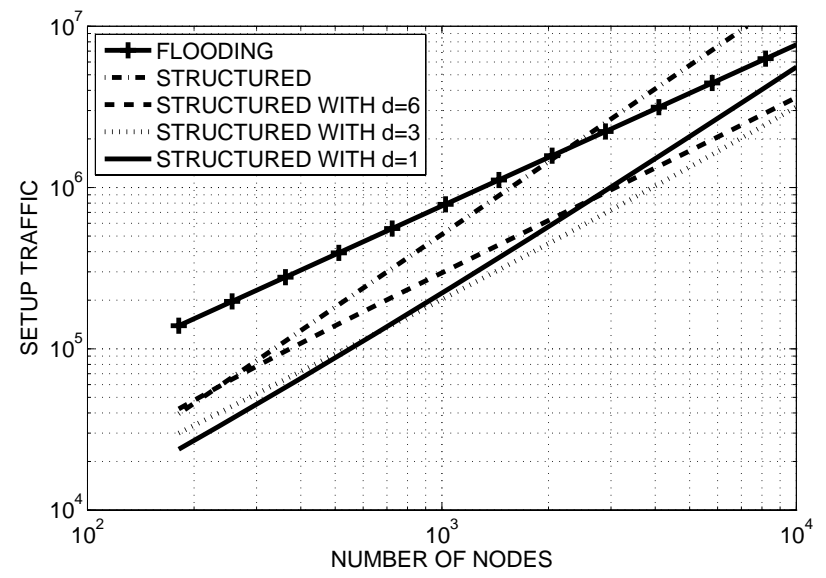

Fig. 6. Setup Traffic vs. Number of Nodes

Figure 7 investigates the influence of the growth of number of items published on the Setup Traffic for $n=1024, N_{R F}=$ 0 and $N_{R U}=1$. Traffic reduction can be found even for reduced number of item such as 10 . As the number of items grow, again the right choice of $d$ becomes an important issue. In this case $d=3$ provide the best result until 200 published items by each node, after that $d=1$ becomes the best design.

The number of packets generated by the Operation Traffic against network size in presented in Figure 8 for just one update operation for $N_{R P}=128, N_{R F}=0$, and $N_{R U}=0$. 


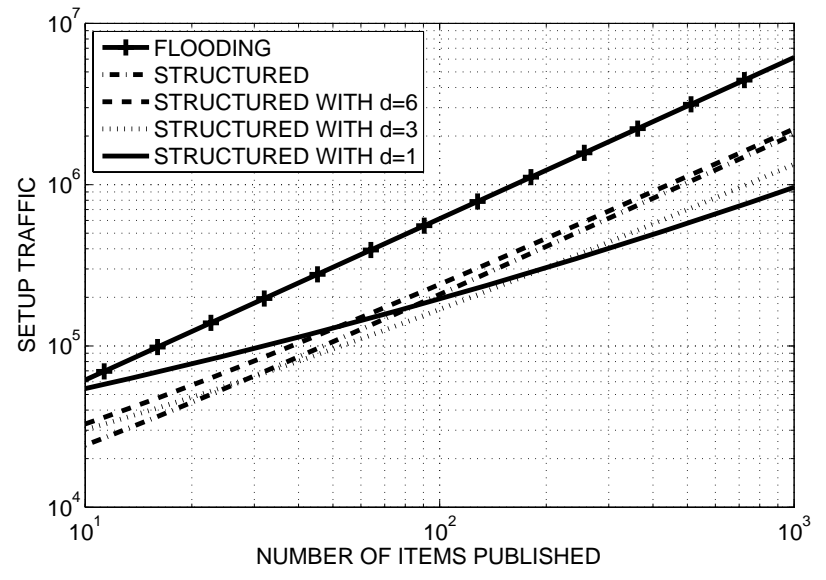

Fig. 7. Setup Traffic vs. Number of Published Items

Almost one order of magnitude reduction can be achieved even for networks below $10^{3}$ nodes. However, as networks grow to $10^{4}$ this advantage over flooding in the Operation Traffic packet count is reduced. A lightpath setup, for example, in proposed scheme would demand multiple searches. On the other hand, nodes in the flooding-based networks hold network global state and no search is needed. However, note that periodic update in flooding systems for information consistency purposes is not accounted in Figure 8.

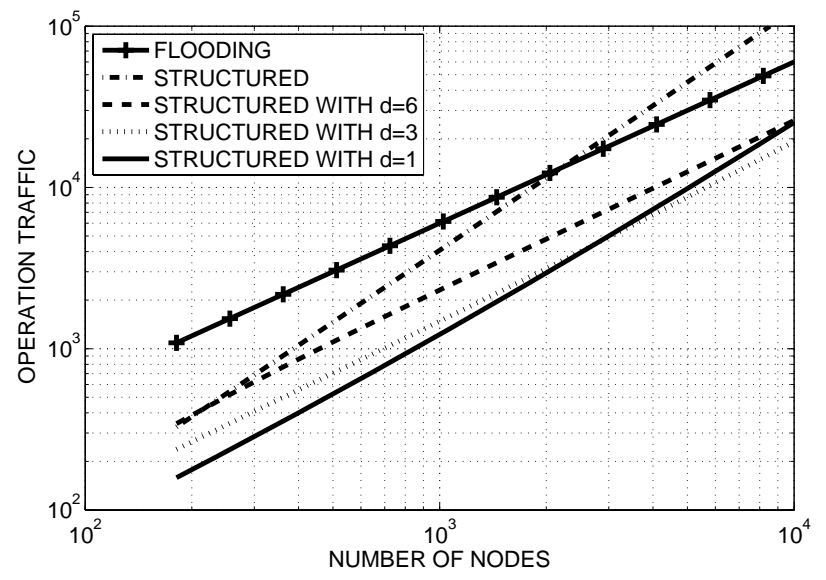

Fig. 8. Operation Traffic vs. Number of Nodes

\section{CONCLUSION}

The amount of information that has to be made available to all nodes in the optical network may demand new strategies of building distributed control planes. The proposed mechanism to resource management brings a new paradigm. By allocating the information on a structured virtual space, a network node can efficiently perform storage of devicestate, enabling information consistency and eliminating the vulnerability to route flapping and information inconsistencies present in flooding based system. The proposed scheme makes possible significant reduction of the number of control packets crossing the network, on startup, without reducing the quantity of information available to nodes. It also allows nodes fetching for just a given resource. Further investigation will be made for dealing with irregular networks and the multiple fetch problem.

\section{REFERENCES}

[1] J. A. D. Rajagopalan, B. Luciani, "Ip over a optical networks: A framework," Network Working Group, vol. IETF, p. RFC 3717, 2004. [Online]. Available: http://tools.ietf.org/html//rfc3717

[2] A. Ganz and I. Chlamtac, "Path allocation access control in fiber optic communication systems," vol. 38, no. 10, pp. 1372-1382, Oct. 1989.

[3] X. Zhang, X. Zhang, and C. Qiao, "Wavelength assignment for dynamic traffic in multi-fiber wdm networks," in Proc. 7th International Conference on Computer Communications and Networks, C. Qiao, Ed., 1998, pp. $479-485$.

[4] B. Ramamurthy and B. Mukherjee, "Wavelength conversion in wdm networking," vol. 16, no. 7, pp. 1061-1073, Sept. 1998.

[5] D. Colle, D. Colle, S. De Maesschalck, M. Pickavet, P. Demeester, M. Jaeger, and A. Gladisch, "Developing control plane models for optical networks," in Proc. Optical Fiber Communication Conference and Exhibit OFC 2002, S. De Maesschalck, Ed., 2002, pp. 757-759.

[6] J. Moy, "Ospf version 2," Network Working Group, vol. IETF, p. RFC 2328, 1998. [Online]. Available: http://tools.ietf.org/html/rfc2328

[7] “Opennap: Open source napster server," http://opennap.sourceforge.net/. [Online]. Available: http://opennap.sourceforge.net/

[8] "Gnutella.com," http://www.gnutella.com/. [Online]. Available: http://www.gnutella.com/

[9] R. R. Plaxton, C. and A. Richa, "Accessing nearby copies of replicated objects in a distributed environment," In Proceedings of the ACM SPAA, vol. Newport, Rhode Island, pp. 311-320, 1997.

[10] A. Rowstron and P. Druschel, "Pastry: Scalable, decentralized object location, and routing for large-scale peer-to-peer systems," Lecture Notes in Computer Science, vol. 2218, pp. 329-??, 2001. [Online]. Available: citeseer.ist.psu.edu/rowstron01pastry.html

[11] I. Stoica, R. Morris, D. Liben-Nowell, D. Karger, M. Kaashoek, F. Dabek, and H. Balakrishnan, "Chord: a scalable peer-to-peer lookup protocol for internet applications," Networking, IEEE/ACM Transactions on, vol. 11, no. 1, pp. 17-32, Feb. 2003.

[12] F. P. H. M. K. R. Ratnasamy, S. and S. Shenker, "A scalable contentaddressable network," In Proc. ACM SIGCOMM, vol. San Diego, CA, August, pp. 161-172, 2001.

[13] K. Y. D. Katz, D. Kompella, "Traffic engineering (te) extensions to ospf version 2," Network Working Group, vol. IETF, p. RFC 3630, 2003. [Online]. Available: http://tools.ietf.org/html/rfc3630

[14] A. Aho and D. Lee, "Hierarchical networks and the lsa n-squared problem in ospf routing," in Global Telecommunications Conference, 2000. GLOBECOM 'O0. IEEE, vol. 1, 27 Nov.-1 Dec. 2000, pp. 397$404 \mathrm{vol} .1$.

[15] C.-X. Chi, D.-W. Huang, D. Lee, and X.-R. Sun, "Lazy flooding: a new technique for signaling in all optical network," in Optical Fiber Communication Conference and Exhibit, 2002. OFC 2002, 17-22 Mar 2002, pp. 551-552.

[16] T. Okazaki, H. Kamada, K. Kinoshita, H. Tode, and K. Murakami, "Efficient flooding control suitable for multiple requests," in Applications and the Internet, 2006. SAINT 2006. International Symposium on, 23-27 Jan. 2006, p. 6pp.

[17] G. Apostolopoulos, "Forwarding plane flooding," in Computers and Communication, 2003. (ISCC 2003). Proceedings. Eighth IEEE International Symposium on, 2003, pp. 963-968vol.2.

[18] K. Khavari, N. Abji, R. Farha, C. Liang, A. Tizghadam, F. Fadaie, and A. Leon-Garcia, "Structured peer-to-peer control plane," in Proc. IEEE International Conference on Communications, vol. 1, June 2006, pp. $48-54$.

[19] M. P. Fardin, M. R. N. Ribeiro, and L. C. Calmon, "Reconfiguração determinística de topologia para geração de característica "small world"," Simpósio Brasileiro de Telecomunicações, 2005.

[20] SECURE HASH STANDARD, 180th ed., Federal Information Processing Standards, August 2002.

[21] H. Zhang, A. Goel, and R. Govindan, "Using the small-world model to improve freenet performance," in INFOCOM 2002. Twenty-First Annual Joint Conference of the IEEE Computer and Communications Societies. Proceedings. IEEE, vol. 3, 23-27 June 2002, pp. 1228-1237vol.3.

[22] M. Li, W.-C. Lee, and A. Sivasubramaniam, "Semantic small world: an overlay network for peer-to-peer search," in Network Protocols, 2004. ICNP 2004. Proceedings of the 12th IEEE International Conference on, 2004, pp. 228-238.

[23] S. Milgram, "The small world problem," Psychology Today, vol. 1, pp. 61-67, 1967.

[24] D. J. Watts and S. H. Strogatz, "Collective dynamics of 'small-world' networks," Nature, vol. 393, pp. 440-442, 1998. 